have had to have been slow enough to enable the gravitational potential energy to be radiated away, a condition which is frequently interpreted in terms of an accretionary period of about 100 million years. The cold accretion theory then usually goes on to say that heating of the Earth by long-lived radioactive isotopes took place later, giving rise to the differentiation which resulted in the formation of the core. In short, core formation may well have taken place some considerable time after accretion (that is, after the formation of the Earth itself) and may even be continuing today.

This is what may be regarded as the basic conventional view of core formation; but there is an important variation on it which, ironically, marks a return to a "hot origin" theory, though in quite a different sense than that intended before the acceptance of accretion. The idea here is that accretion took place too rapidly for the gravitational potential energy to be radiated away effectively, that the accretion temperature was thus high, and that the conditions necessary for core formation obtained more or less at the time of accretion. The first proponent of this view was probably Ringwood (Geochim. Cosmochim. Acta, 20, $241 ; 1960)$ who supported it on geochemical grounds, although it was later taken up by Hanks and Anderson (Phys. Earth Planet. Interiors, 2, $19 ; 1969)$ and. more recently, received experimental support from Oversby and Ringwood (Nature, 234, $463 ; 1971$ ).

But whether the core formed during, or later than, accretion, there has been little suggestion so far that the mixture of iron and silicates from which the Earth accreted was anything but homogeneous; and it is this assumption of homogeneity which effectively defines what is now recognized as the conventional view of core formation. In this connexion the work of Elsasser (Earth Science and Meteorites, North-Holland, Amsterdam; 1963) may be regarded as representative.

In considering the early history of the Earth, Elsasser

\section{A Chemical Curiosity}

IT is always interesting to find chemical curiosities and it is often in the course of kinetic studies that these turn up. Examples occur of concurrent or consecutive reactions which demonstrate, because of their relative rates or efficiencies, the build-up and decay of intermediate species. This is particularly attractive when an obvious colour change simplifies the observation and enables the observer to appreciate visually a new "art form".

$A$ recent addition to the gallery is a reaction discussed by Field and Noyes on page 390 of this issue of Nature. This involves the reaction of bromate ion on malonic acid catalysed by cerium ions. Depending on the conditions of experiment, oscillations in the cerous/ceric ratio can occur either as a function of time or space and, as might be expected, this is accomplished by a series of interconnected reactions. The authors of the letter promise a subsequent discussion of the implications of this type of chemical sequence which may even provide an alternative to the familiar egg timer.-From a Correspondent. started with an Earth which accreted cold and in which the material was uniformly distributed. The principal feature of such a model is that the melting point curve of the silicates rises much more steeply with depth than the actual temperature, which implies an appreciable rise in the viscosity of the silicates with depth. As the Earth is later heated by radioactive decay, the outer layers are the first to become soft enough to allow iron to move towards the centre. At greater depths, however, the sinking of the iron is slowed down by the increased viscosity, the iron forms a coherent layer which is gravitationally unstable, and the large drops which result fall rapidly to the centre forming a core. Elsasser's estimate of the time taken to form a core slightly smaller than the present one was about $10^{8}$ years. Another, equally well known, model beginning with a homogeneous Earth was that of Birch (J. Geophys. Res., 70, 6217 ; 1965).

With Orowan (Nature, 222, $867 ; 1969$ ), however, a new idea emerged (albeit within the framework of a model which was perhaps less in keeping with mainstream thinking); namely, that the Earth may have accreted inhomogeneously right from the start. Orowan's point was that, even at low temperatures, iron is plastic-ductile as long as its carbon content does not vastly exceed that of meteorites. As a result, colliding metal particles may be expected to cohere because they can absorb kinetic energy by plastic deformation and can thus combine by cold or hot welding. Silicates, on the other hand, being brittle. break up on collision except at near melting point temperatures. The idea is then that the Earth's accretion began with metallic particles. Once sufficiently large, the growing Earth would then collect non-metallic particles by embedding them in ductile material and later by gravitational attraction. In other words, according to this view, the Earth formed with a metal core already partially differentiated, core formation and Earth accretion were thus simultaneous, and later core formation by differentiation was unnecessary.

The same year, Turekian and Clark (Earth Planet. Sci. Lett., 6, 346; 1969) also proposed an inhomogeneous model, but based on rather different principles. Their idea was that as the primitive solar nebula cooled, elements and compounds would condense in the order of increasing vapour pressure, an order defined by Larimer (Geochim. Cosmochim. Acta, 31, $1215 ;$ 1967) as iron and nickel, magnesium and iron silicates, alkali silicates, metals such as $\mathrm{Ag}, \mathrm{Ga}$ and $\mathrm{Cu}$, iron sulphide, and metals such as $\mathrm{Hg}, \mathrm{Tl}, \mathrm{Pb}$, In and $\mathrm{Bi}$. In general terms such an order agrees with the stratification in the Earth usually attributed to differentiation. Thus, according to this model, condensed iron-nickel would first form the core which, as in Orowan's model, would become the nucleus for the deposition of silicate mantle.

There is little doubt that such inhomogeneous models need to be tested, if only because, as Ringwood (Geochim. Cosmochim. Acta, 30, $41 ; 1966)$ pointed out some years ago, there are a number of difficulties with the conventional model. On the other hand, as Anderson and Hanks point out in this issue of Nature, the Turekian-Clark model has also hitherto been stuck with a problemnamely, that there is apparently not enough energy available to melt the outer core either during or after accretion. By eliminating this problem, Anderson and Hanks have now made the idea of initial inhomogeneity that much more attractive.-P. J. S. 